International Journal of Electrical Engineering and Technology (IJEET)

Volume 11, Issue 4, June 2020, pp. 108-113, Article ID: IJEET_11_04_012

Available online at https://iaeme.com/Home/issue/IJEET? Volume $=11 \&$ Issue $=4$

ISSN Print: 0976-6545 and ISSN Online: 0976-6553

DOI: https://doi.org/10.34218/IJEET.11.4.2020.012

\title{
DESIGN AND DEVELOPMENT OF A NOVEL QUALITY ASSURANCE REGRESSION INFRASTRUCTURE
}

\author{
Ganesh Govind* \\ Department of Electronics and Communication Engineering, \\ RV College of Engineering, Bengaluru, India
}

Kiran V

Associate Professor, Department of Electronics and Communication Engineering, RV College of Engineering, Bengaluru, India

John Alwyn

Principal Application Engineer, ANSYS, Inc Bengaluru (Marathahalli), India

*Corresponding Author Email: ganeshg.ec16@rvce.edu.in

\begin{abstract}
The VLSI design complexity has increased exponentially over the last few years and the transition to $7 / 5 \mathrm{~nm}$ and beyond is a big jump. There is 5 times increase in grid complexity from $16 \mathrm{~nm}$ to $7 \mathrm{~nm}$ designs and the grids have more than 10 billion nodes. Design robustness, reliability, power integrity issues are a primary concern to be solved which has led to the development of a software on big data platforms which can perform multi-dimensional analysis on the design. For these and other software products, there are several release cycles in a year to meet customer requirements. With each release, build quality needs to be verified. Therefore, a modular and a robust tool to check release quality is required. The infrastructure developed in this project will regress several testcases and data mine several parameters from each run and compare it against a golden reference. The results from comparison will then be tabulated and reviewed to ensure there are no issues in product build. This paper focuses on building a quality assurance tool, to be used for the comparison of various parameters and which is adaptable in nature i.e. it can support quick and easy addition of new parameters in the future as per the requirement of any product.
\end{abstract}

Key words: Quality Assurance (QA), Regression Infrastructure, Adaptive Quality Assurance Tool. 
Cite this Article: Ganesh Govind, Kiran V and John Alwyn, Design and Development of a Novel Quality Assurance Regression Infrastructure, International Journal of Electrical Engineering and Technology, 11(4), 2020, pp. 108-113. https://iaeme.com/Home/issue/IJEET?Volume=11\&Issue $=4$

\section{INTRODUCTION}

During a product's life cycle, software undergoes enhancement, bug fixes based on customer requests. By reviewing various parameters from regression case runs, one can assess if the parameter changes are related to software change and assess if the measured magnitude of this change is expected. This helps ensure that the product delivered to customer does not break existing simulation methodology used by customer and saves valuable design cycle time. One of the important areas of research includes optimizing the QA tools. Novel ideas in this area such as detecting the code which is pseudo-tested, detecting the low fault risk code have been discussed in [1]. A tool that incorporates and supports the implementation of various practices for the QA with a detailed description and all the validation checks done to the tool are dealt with in [2].

To illustrate and validate such tools an algorithm (Software Reliability Growth Modeling (SRGM) has been proposed and the major cases of use, which include original defect, data given by 2 development projects in the telecom industry are used in [3]. A vivid picture regarding the application of Software Quality Metrics (SQM) in the quality life cycle and the significance of SQM, mainly concentrating on time, which will significantly influence the quality of the final product build is portrayed in [4].

The challenges related to QA in software ecosystems and the possible approaches have been proposed. The method used is a systematic literature mapping in [5]. The software quality standards and software QA and their importance is dealt with. It is shown that the quality of every software needs to be guaranteed in par with the global quality standards. Various test mechanisms to test the quality have also been dealt with in [6].

A new 3C approach is proposed: CM (Continuous Measurement), CI (Continuous Improvement) have been added to CI (Continuous Integration) for an Agile QA in [7]. Any standard code is complex and hence usually software containing errors is released. To overcome these errors systematically, inspection methods are adopted. The software design will definitely become easier when computer support is taken and the process becomes more effective as described in [8].

Many agile methods may produce the final build of the software quicker, but in order to make sure that all the requirements in terms of quality is met is also of importance. A comparison of the waterfall model and the various processes that are agile is done and finally concluded that methods which are agile are achieving greater quality in terms of the final software build in spite of adding extra pressure in time and creating requirements which are unstable are described in [9]. The lasting impact of strategical decisions on the resulting software quality is important. Quantification always takes place making use of enough software metrics. The impact of the QA strategy on the quality of the product is identified on the basis of data from several sources including industry projects using statistics in [10]. The Ansys Totem-SC product was studied in detail in order to analyze how the parameters are extracted and dumped for QA development [11].

\section{METHODOLOGY}

The QA infrastructure developed in this project uses ANSYS Totem-SC (SeaScape) for testing and improving the model by collecting the various releases and performing several checks. The methodology of the project is shown in Fig. 1. 
The various regression testcases are passed to ANSYS Totem-SC for analysis which runs these cases and translates the GDS (Graphic Data System) to LEF (Library Exchange Format) and DEF (Design Exchange Format) files which are used as inputs to create the design view. $\mathrm{RC}$ network of Layout data available in design view is created in extract view.

The two views namely design view and extract view upon extraction have various checks to be performed for several parameters to test the infrastructure. The methodology involves designing and developing an infrastructure to capture all the parameters (such as Die Area, mesh resistance estimates etc.) and compare these with those obtained in another version of Totem-SC. The infrastructure is developed completely in Python 2.7. The various parameters mined in the 2 views for testing the infrastructure are:

- Design View Parameters- Die Area, Power Nets, Ground Nets, Signal Nets, Instance count, Switch Instance Count, transistor pin snapping report bounding box, transistor pin snapping report pin snapping layer, transistor pin snapping error, DEF pins, Switch model file (includes resistance, capacitance of the pin taps).

- Extract view parameters- summarized as mesh resistance estimate per device and summary of node counts, resistance and capacitance counts.

Apart from these parameters, the performance statistics is extracted which gives the time and memory consumed for generating each view. These parameters are extracted and used to test the infrastructure. Later, once the infrastructure is developed, any number of new parameters can be easily added.

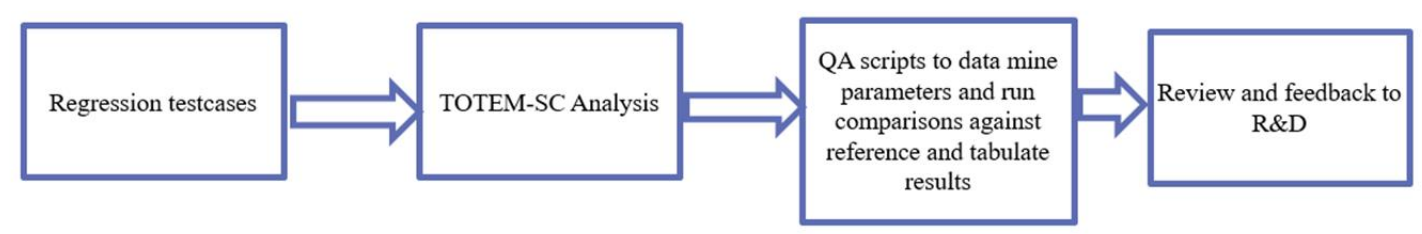

Figure 1 Entire Methodology of the Project

\section{QUALITY ASSURANCE REGRESSION INFRASTRUCTURE}

\subsection{Design of the Infrastructure}

The infrastructure designed and developed comprises of 4 main scripts as follows: -

- main code script - Contains the parameter specific dump and compare functions.

- dump parameters script- Contains functions to mine the view related files and dump the parameter DataFrames into the disk as csv files. This file calls the dump functions from the main script.

- path builder script- Contains modules to extract the view related files' HTML clickable versions and are returned to compare on call. These files are used to display when a case related to a parameter passes.

- compare script- Reads the DataFrames from the csv files in the disk and calls the compare modules from the main code script to perform the parameter specific comparison. It also contains modules which use the path builder script's modules to get view related files for displaying when a case passes. It finally generates parameter and case specific pass/fail/error HTML pages which are linked to a summary HTML page to display the results.

To store and handle all the data, the DataFrame object from the pandas open source library is used in this paper. This is efficient in data preparation and analysis especially in case 
of large data. There are readily available inbuilt library methods to handle the DataFrame objects which include functions to dump the DataFrames to the disk in csv format, to dump the DataFrame to an HTML page etc.

\subsection{Modular Design of the Code}

A config file is taken as the input to the comparison run and this file provides the paths to the case directories where the necessary parameter related files are dumped in 2 modes. In mode 1 , the parent directory is provided for both the versions where all the case-wise directories reside. In mode 2, the case-wise directories corresponding to the respective versions are passed directly using a file. The code is written in an efficient and modular fashion as depicted in Fig. 2. With the code to create the design and the extract view, a call is made to the script which dumps all the parameters to the disk in csv format by calling in turn all the individual dump functions for each parameter. Once all the csv files are dumped, a comparison script is called which in turn calls a function to build all the paths to the csv files in the disk and also to the view dump files which will be displayed when the case passes.

The comparison script is modular in itself and calls separate functions to create the necessary HTML pages and to compare all the individual parameters. All these dump functions and comparison functions are imported into the comparison script and dump script and is stored separately as the main code. The main code will require just the addition of a dump and a compare function when a new parameter is to be added. Hence, the design is made extendable to the parameters that will be added later.

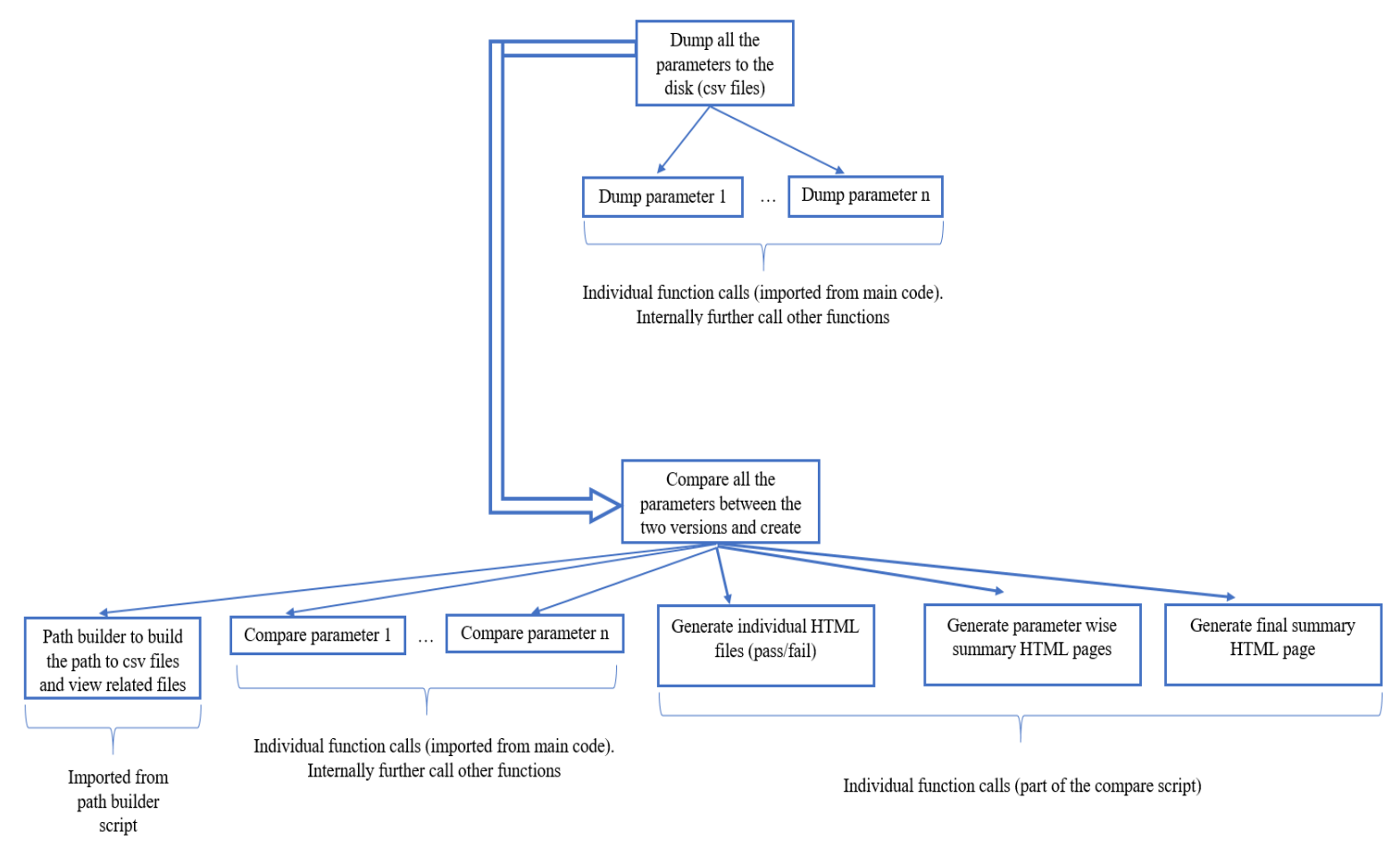

Figure 2 Modular design of the code

\section{RESULTS}

Various cases were used for testing the developed QA tool. These cases involved all possible varieties including any corner cases necessary. A final summary page for the design and analysis views (as shown in Fig. 3) is dumped with the current date and time of the QA run for ease of search. 
This page contains the final summary results for version to version comparison for various cases between the Totem-SC releases. This page is further linked to the summary pages of the respective parameters with pass or fail conditions. On clicking on the count of pass/fail/error for any parameter, another page with the necessary details showing the pass/fail/error summary.

- In case of a pass for a particular case, the file paths dumped in the system are given and can be opened to verify the contents.

- In case of a fail (even if a single parameter fails, it is seen as a fail case), a parameterwise customized fail detail page is developed which displays the fail case and the necessary details regarding the details in both the files in the 2 versions which caused the fail.

- The error cases include the cases for which necessary input files or views were not found or the modules could not be executed correctly.

These parameter wise case level descriptions are useful in debugging the errors and improving the software in the coming builds.

\section{Ansys}

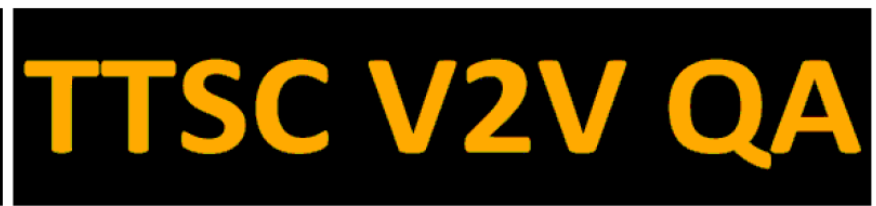

\begin{tabular}{|c|c|c|c|c|c|c|}
\hline & Parameter & No. Of Cases & No. of Error Cases & Pass Count & Fail Count & Details \\
\hline 0 & Performance Statistics & 3 & $\underline{0}$ & $\underline{0}$ & $\underline{3}$ & Description \\
\hline 1 & Die Area & 3 & $\underline{1}$ & $\underline{2}$ & $\underline{0}$ & Description \\
\hline 2 & Gated Power Nets & 3 & $\underline{2}$ & $\underline{1}$ & $\underline{0}$ & Description \\
\hline 3 & Gated Ground Nets & 3 & $\underline{2}$ & $\underline{1}$ & $\underline{0}$ & Description \\
\hline 4 & Switch Model File & 3 & 2 & $\underline{1}$ & $\underline{0}$ & Description \\
\hline 5 & Instance Count & 3 & $\underline{0}$ & $\underline{3}$ & $\underline{0}$ & Description \\
\hline 6 & Switch Instance Count & 3 & $\underline{0}$ & $\underline{3}$ & $\underline{0}$ & Description \\
\hline 7 & DEF pins & 3 & $\underline{1}$ & $\underline{2}$ & $\underline{0}$ & Description \\
\hline 8 & xtor pin snapping error & 3 & $\underline{3}$ & $\underline{0}$ & $\underline{0}$ & Description \\
\hline 9 & clamp info file & 3 & $\underline{3}$ & $\underline{0}$ & $\underline{0}$ & Description \\
\hline 10 & xtor pin snapping report pin bbox & 3 & $\underline{1}$ & $\underline{2}$ & $\underline{0}$ & Description \\
\hline 11 & Power Nets & 3 & $\underline{0}$ & $\underline{3}$ & $\underline{0}$ & Description \\
\hline 12 & Ground Nets & 3 & $\underline{0}$ & $\underline{3}$ & $\underline{0}$ & Description \\
\hline 13 & xtor pin snapping report pin psl & 3 & $\underline{1}$ & $\underline{2}$ & $\underline{0}$ & Description \\
\hline 14 & Switch xtor grouping & 3 & $\underline{2}$ & $\underline{1}$ & $\underline{0}$ & Description \\
\hline 15 & disconnected_instance_pins & 3 & $\underline{0}$ & $\underline{3}$ & $\underline{0}$ & Description \\
\hline 16 & Circuit_stats & 3 & $\underline{0}$ & $\underline{2}$ & 11 & Description \\
\hline
\end{tabular}

Figure 3 Final summary page containing the results for 3 cases run between Ansys Totem-SC Versions

\section{CONCLUSION}

Hence, this Quality Assurance tool can be used to quickly and efficiently run any number of cases between product releases of a software. The tool developed is efficient and modular and can be used to add any number of parameters in the future with ease (by creating the necessary functions to dump and compare for each parameter). The entire infrastructure is designed and only the parameter related functions must be added to the code for adding a new parameter related to any software.

\section{ACKNOWLEDGEMENT}

This research was supported by RV College of Engineering and Ansys, Inc. 
Design and Development of a Novel Quality Assurance Regression Infrastructure

\section{REFERENCES}

[1] D. Winkler, S. Biffl, D. Mendez, and J. Bergsmann, Eds., Software Quality: Quality Intelligence in Software and Systems Engineering. Springer International Publishing, 2020. doi: 10.1007/978-3-030-35510-4.

[2] S. Ibarra and M. Munoz, "Support tool for software quality assurance in software development," in 2018 7th International Conference On Software Process Improvement (CIMPS), IEEE, Oct. 2018. doi:10.1109/cimps.2018.8625617.

[3] K. Okumoto, R. Mijumbi, and A. Asthana, "Software quality assurance," in Telecommunication Networks - Trends and Developments, IntechOpen, Feb. 2019. doi: 10.5772/intechopen.79839.

[4] J. Vijay, G. Chand, and H. Done, "Software quality metrics in quality assurance to study the impact of external factors related to time," International Journal of Advanced Research in Computer Science and Software Engineering, vol. 7, no. 1, pp. 221-224, Jan. 2017. doi: 10.23956/ijarcsse/v6i11/0114.

[5] J. Axelsson and M. Skoglund, "Quality assurance in software ecosystems: A systematic literature mapping and research agenda," Journal of Systems and Software, vol. 114, pp. 69-81, Apr. 2016. doi: 10.1016/j.jss.2015.12.020.

[6] P. Shen, X. Ding, W. Ren, and C. Yang, "Research on software quality assurance based on software quality standards and technology management," in 2018 19th IEEE/ACIS International Conference on Software Engineering, Artificial Intelligence, Networking and Parallel/Distributed Computing (SNPD), IEEE, Jun. 2018. doi: 10.1109/snpd.2018.8441142.

[7] A. Janus, R. Dumke, A. Schmietendorf, and J. Jager, "The 3c approach for agile quality assurance," in 2012 3rd International Workshop on Emerging Trends in Software Metrics (WETSoM), IEEE, Jun. 2012. doi: 10.1109/wetsom.2012. 6226998.

[8] D. Parnas and M. Lawford, "The role of inspection in software quality assurance," IEEE Transactions on Software Engineering, vol. 29, no. 8, pp. 674-676, Aug. 2003. doi: 10.1109/tse.2003.1223642.

[9] M. Huo, J. Verner, L. Zhu, and M. Babar, "Software quality and agile methods," in Proceedings of the 28th Annual International Computer Software and Applications Conference, 2004. COMPSAC 2004., IEEE. doi: 10.1109/cmpsac.2004.1342889.

[10] C. Kugler, S. Kowalewski, J. Richenhagen, R. Maquet, and A. Schloßer, "Metricsbased strategies for quality assurance of automotive embedded software," in 17. Internationales Stuttgarter Symposium, Springer Fachmedien Wiesbaden, 2017, pp. 711- 730. doi: 10.1007/978-3-658-16988-6_56.

[11] Ansys Totem-SC User Manual 